\title{
IRREDUCIBLE QUASIFINITE MODULES OVER A CLASS OF LIE ALGEBRAS OF BLOCK TYPE*
}

\author{
HONGJIA $\mathrm{CHEN}^{\dagger}$, XIANGQIAN GUO ${ }^{\ddagger}$, AND KAIMING ZHAO
}

\begin{abstract}
For any nonzero complex number $q$, there is a Lie algebra of Block type, denoted by $\mathcal{B}(q)$. In this paper, a complete classification of irreducible quasifinite modules is given. More precisely, an irreducible quasifinite module is a highest weight or lowest weight module, or a module of intermediate series. As a consequence, a classification for uniformly bounded modules over another class of Lie algebras, the semi-direct product of the Virasoro algebra and a module of intermediate series, is also obtained. Our method is conceptional, instead of computational.
\end{abstract}

Key words. Block type algebra, Virasoro algebra, quasifinite module.

AMS subject classifications. 17B10, 17B20, 17B65, 17B66, $17 \mathrm{~B} 68$.

1. Introduction. Because of wide applications in many mathematics and physics branches, the representation theory of the Virasoro algebra has been extensively studied ([CP], $[\mathrm{KR}])$. Recently, many authors investigated Harish-Chandra modules (or quasifinite weight modules) for several infinite Lie algebras related to the Virasoro algebra, for example, generalized Virasoro algebras, the Heisenberg-Virasoro algebra, the loop-Virasoro algebra, truncated Virasoro algebras, the algebra $W(2,2)$, Schrödinger-Virasoro algebras, the Virasoro-like algebra, q-analog of Virasoro-like algebras, Block type algebras $\mathcal{B}$ and $\mathcal{B}(q)$ with $q$ a nonzero complex number. In particular, irreducible Harish-Chandra modules for the Virasoro algebra, generalized Virasoro algebras, the Heisenberg-Virasoro algebra, the loop-Virasoro algebra, truncated Virasoro algebras, the algebra $W(2,2)$, and Block algebra $\mathcal{B}$ are completely classified (See [M, GLZ1, GLZ2, LZ1, LZ2, S1, S2, S3]). For other algebras such as SchrödingerVirasoro algebras, the Virasoro-like algebra, q-analog of Virasoro-like algebras, Block type algebras $\mathcal{B}(q)$, irreducible Harish-Chandra modules (or quasifinite modules) are divided into two classes: (generalized) highest or lowest weight modules and uniformly bounded modules (See [LS, LT1, LT2, SXX1, WT]). Unfortunately, the structure for the uniformly bounded modules is unclear. In this paper, we solve this problem for Lie algebras $\mathcal{B}(q)$ with nonzero complex numbers $q$.

Let us first recall the definition for the Lie algebras $\mathcal{B}(q)$.

Denote by $\mathbb{Z}, \mathbb{N}, \mathbb{Z}_{+}$and $\mathbb{C}$ the sets of integers, positive integers, nonnegative integers and complex numbers respectively. For any complex number $q$, the Lie algebra $\mathcal{B}(q)$ has a basis $\left\{L_{m, i}, C \mid m \in \mathbb{Z}, i \in \mathbb{Z}_{+}\right\}$over $\mathbb{C}$ subject to the following Lie brackets

$$
\begin{gathered}
{\left[L_{m, i}, L_{n, j}\right]=(n(i+q)-m(j+q)) L_{m+n, i+j}+\delta_{m+n, 0} \delta_{i+j, 0} \frac{m^{3}-m}{12} C} \\
{\left[C, L_{m, i}\right]=0}
\end{gathered}
$$

*Received July 6, 2012; accepted for publication July 5, 2013.

†School of Mathematical Sciences, University of Science and Technology of China, Wu Wen Tsun Key Laboratory of Mathematics, Chinese Academy of Science, Hefei 230026, Anhui, P. R. China (hjchen@ustc.edu.cn).

${ }^{\ddagger}$ Corresponding author. School of Mathematics and Statistics, Zhengzhou University, Zhengzhou 450001, Henan, P. R. China (guoxq@zzu.edu.cn).

$\S$ Department of Mathematics, Wilfrid Laurier University, Waterloo, ON, Canada N2L 3C5 (kzhao@wlu.ca); and College of Mathematics and Information Science, Hebei Normal (Teachers) University, Shijiazhuang 050016, Hebei, P. R. China. 
where $m, n \in \mathbb{Z}, i, j \in \mathbb{Z}_{+}$.

Note that the Lie algebras $\mathcal{B}(q)$ are in fact subalgebras of some very special cases of generalized Block algebras studied in [DZ], the Lie algebra $\mathcal{B}(0)$ is a half part of the well-known Virasoro-like algebra, and $\mathcal{B}(1)$ is the Block type Lie algebra studied in [WT].

The paper is organized as follows. In Sect.2, we determine all ideals of $\mathcal{B}(q)$ and construct all irreducible uniformly bounded modules in a different approach from that in [SXX1]. In Sect.3, we prove that any nontrivial irreducible uniformly bounded module for $\mathcal{B}(q)$ with $q \neq 0$ is of intermediate series. Thus we give a complete classification for irreducible quasifinite modules over $\mathcal{B}(q)$. We also classify irreducible uniformly bounded modules for another class of Lie algebras, the semi-direct product of the Virasoro algebra and one of its modules of intermediate series.

Throughout this paper, $q$ is always assumed to be a fixed nonzero complex number unless specified otherwise. For any subset $S$ in $\mathbb{C}$, denote $S^{*}=S \backslash\{0\}$. All vector spaces and (Lie) algebras are over $\mathbb{C}$. For a Lie algebra $\mathcal{G}$, we denote its universal enveloping algebra by $U(\mathcal{G})$. For $a \in \mathbb{C}$ and $S \subseteq \mathbb{C}, \delta_{a, S}=1$ if $a \in S$ and 0 otherwise.

2. Constructing modules over $\mathcal{B}(q)$. The algebra $\mathcal{B}(q)$ can be realized in $\mathbb{C}\left[x, x^{-1}\right] \otimes t^{q} \mathbb{C}[t] \oplus \mathbb{C} C$ as follows: For any $m \in \mathbb{Z}$ and $f(t)=\sum_{i=0}^{d} a_{i} t^{i} \in \mathbb{C}[t]$, we denote $x^{m} t^{q} f(t)=\sum_{i=0}^{d} a_{i} L_{m, i}$. In particular, $x^{m} t^{q+i}=L_{m, i}$ for all $m \in \mathbb{Z}$ and $i \in$ $\mathbb{Z}_{+}$, where we consider $t^{q+i}$ as a formal power of the indeterminant $t$. Consequently, the Lie bracket of $\mathcal{B}(q)$ in $(1.1)$ can be rewritten as

$$
\begin{aligned}
{\left[x^{m} f(t), x^{n} g(t)\right]=} & x^{m+n} t^{1-q}\left(n f^{\prime}(t) g(t)-m f(t) g^{\prime}(t)\right) \\
& +\delta_{m+n, 0} \frac{m^{3}-m}{12} \operatorname{Res}\left(t^{-2 q-1} f(t) g(t)\right) C,
\end{aligned}
$$

for $m, n \in \mathbb{Z}$ and $f(t), g(t) \in t^{q} \mathbb{C}[t]$, where $f^{\prime}(t)$ is the usual derivative of $f(t)$ and $\operatorname{Res} f(t)$ is the residue of $f(t)$, namely the coefficient of $t^{-1}$ in $f(t)$. In what follows, we will use these two different notations for $\mathcal{B}(q)$ alternatively and freely.

The Lie algebra $\mathcal{B}(q)$ has a natural $\mathbb{Z}$-gradation $\mathcal{B}(q)=\oplus_{m \in \mathbb{Z}} \mathcal{B}(q)_{m}$ with

$$
\mathcal{B}(q)_{m}=\operatorname{span}_{\mathbb{C}}\left\{L_{m, i} \mid i \in \mathbb{Z}_{+}\right\} \oplus \delta_{m, 0} \mathbb{C} C .
$$

This gradation is with respect to the eigenvalues of $\operatorname{ad}\left(L_{0,0}\right)$.

It is easy to see that $\mathcal{B}(q)$ is perfect, i.e., $[\mathcal{B}(q), \mathcal{B}(q)]=\mathcal{B}(q)$ if and only if $-2 q \notin \mathbb{N}$. For convenience, denote $\mathcal{L}=[\mathcal{B}(q), \mathcal{B}(q)] /\left(\mathbb{C} C \oplus \delta_{-q, \mathbb{N}} \mathbb{C} L_{0,-q}\right)$, i.e., centerless algebra of $[\mathcal{B}(q), \mathcal{B}(q)]$. We use the same notation $L_{m, i}$ for its image in $\mathcal{L}$. For any ideal $I$ of $[\mathcal{B}(q), \mathcal{B}(q)]$, we denote by $\widetilde{I}$ the image of $I$ in $\mathcal{L}$. Note that all the algebras $\mathcal{B}(q),[\mathcal{B}(q), \mathcal{B}(q)], \mathcal{L}$ and their ideals are $\mathbb{Z}$-graded with respect to the gradation $(2.2)$.

The algebra $\mathcal{B}(q)$ has a series of ideals, that is,

$$
I_{k}=\operatorname{span}_{\mathbb{C}}\left\{x^{m} t^{q+i} \mid m, i \in \mathbb{Z}, i \geqslant k\right\}+\delta_{k, 0} \mathbb{C} C, \quad \forall k \in \mathbb{Z}_{+} .
$$

In case that $-2 q \in \mathbb{N}$, we have some other ideals of $\mathcal{B}(q)$, i.e.,

$$
I_{k}^{\prime}=\operatorname{span}_{\mathbb{C}}\left\{x^{m} t^{q+i} \mid m, i \in \mathbb{Z}, i \geqslant k,(m, i) \neq(0,-2 q)\right\}+\delta_{k, 0} \mathbb{C} C, \quad \forall k \in \mathbb{Z}_{+} .
$$

We shall identify $I_{k}^{\prime}$ and $I_{k}$ if $-2 q \notin \mathbb{N}$. For any $k \in \mathbb{Z}_{+}, I_{k}^{\prime}$ is an ideal of $[\mathcal{B}(q), \mathcal{B}(q)]$ and $\widetilde{I_{k}^{\prime}}$ an ideal of $\mathcal{L}$.

Let $\mathcal{I}$ be an ideal of $\mathcal{L},[\mathcal{B}(q), \mathcal{B}(q)]$ or $\mathcal{B}(q)$. A polynomial $f=\sum_{i \in \mathbb{Z}_{+}} a_{i} t^{i} \in \mathbb{C}[t]$ is called strange for $\mathcal{I}$ if there exists $m \in \mathbb{Z}$ such that $x^{m} t^{q} f(t) \in \mathcal{I}$ but $x^{n} t^{q+i} \notin \mathcal{I}$ 
for all $n \in \mathbb{Z}$ and all $i$ with $a_{i} \neq 0$. If there is a strange polynomial $f(t)$ for $\mathcal{I}, \mathcal{I}$ is called a strange ideal. None of the ideals defined above is strange. Indeed, we will see from the next lemma that there are no strange ideals unless $q=-1$.

When $q=-1$, we have the following ideals for both $\mathcal{B}(q)$ and $[\mathcal{B}(q), \mathcal{B}(q)]$

$$
J_{a}=I_{3} \oplus \sum_{m \in \mathbb{Z}} \mathbb{C} x^{m} t^{q}\left(t+a m t^{2}\right), \forall a \in \mathbb{C} .
$$

Note that $J_{a}$ is a strange ideal of $\mathcal{B}(-1)$ and $[\mathcal{B}(-1), \mathcal{B}(-1)]$, while $\widetilde{J_{a}}$ is a strange ideal of $\mathcal{L}$ for any $a \neq 0$. Now we can determine all ideals of $\mathcal{B}(q),[\mathcal{B}(q), \mathcal{B}(q)]$ and $\mathcal{L}$.

Lemma 1. Let $C^{\prime}=\delta_{-q, \mathbb{N}} L_{0,-q}$ and $C^{\prime \prime}=\delta_{-2 q, \mathbb{N}} L_{0,-2 q}$.

(1). All ideals of $\mathcal{L}$ are: $0, \widetilde{I_{k}^{\prime}}$ for any $k \in \mathbb{Z}_{+}$, and $\delta_{q,-1} \widetilde{J_{a}}$ for any $a \in \mathbb{C}$.

(2). All ideals of $[\mathcal{B}(q), \mathcal{B}(q)]$ are: $K, \delta_{q,-1} J_{a}$ and $\delta_{q,-1}\left(J_{a}+\mathbb{C} C\right)$ for any $a \in \mathbb{C}$, and $I_{k}^{\prime}+K$ for any $k \in \mathbb{Z}_{+}$, where $K$ is a subspace of $\mathbb{C} C \oplus \mathbb{C} C^{\prime}$.

(3). Any ideal of $\mathcal{B}(q)$ is one of the ideals in (2) or $I_{k}^{\prime}+K$ for $-2 q-k \in \mathbb{Z}_{+}$, where $K$ is a subspace of $\mathbb{C} C \oplus \mathbb{C} C^{\prime} \oplus \mathbb{C} C^{\prime \prime}$.

Proof. Suppose $\mathcal{I}$ is a nonzero ideal of $\mathcal{L}$. Let $k \in \mathbb{Z}_{+}$be such that $\mathcal{I} \subseteq \widetilde{I}_{k}^{\prime}$ but $\mathcal{I} \nsubseteq \widetilde{I_{k+1}^{\prime}}$. Note that $\mathcal{I}=\bigoplus_{m \in \mathbb{Z}} \mathcal{I}_{m}$, where $\mathcal{I}_{m}=\left\{x \in \mathcal{I} \mid\left[L_{0,0}, x\right]=m q x\right\}$. If $L_{m, i} \in \mathcal{I}$ for some $m \in \mathbb{Z}, i \in \mathbb{Z}_{+}$with $(m, i) \neq(0,-q),(0,-2 q)$ and $(q, i) \neq(-1,1)$, then we can deduce that $L_{n, j} \in \mathcal{I}$ for all $n \in \mathbb{Z}$ and $j \geqslant i$ with $(n, j) \neq(0,-2 q)$.

First suppose that $\mathcal{I}$ is not a strange ideal. There is $L_{m, k} \in \mathcal{I}$. By the Lie bracket (2.1), if $q \neq-1$ or $k \neq 1$, we have $\widetilde{I_{k}^{\prime}} \subseteq \mathcal{I}$, i.e., $\mathcal{I}={\widetilde{I_{k}^{\prime}}}^{\prime}$. If $q=-1$ and $k=1(m \neq 0$ in this case), then we have $\widetilde{I}_{3}^{\prime} \oplus \sum_{m \in \mathbb{Z}^{*}} \mathbb{C} L_{m, 1} \subseteq \mathcal{I}$. If there is $n \in \mathbb{Z}^{*}$ such that $L_{n, 2} \in \mathcal{I}$, then $\widetilde{I_{1}^{\prime}} \subseteq \mathcal{I}$, i.e., $\mathcal{I}=\widetilde{I}_{1}^{\prime}$, otherwise $\mathcal{I}={\widetilde{I_{3}^{\prime}}}_{3} \oplus \sum_{m \in \mathbb{Z}^{*}} \mathbb{C} L_{m, 1}=\widetilde{J_{0}}$.

Now suppose that $\mathcal{I}$ is a strange ideal. For any $f(t)=\sum_{i \in \mathbb{Z}_{+}} a_{i} t^{i} \in \mathbb{C}[t]$, denote by $\ell(f)$ the number of nonzero $a_{i}$. By the above discussion, we can take a strange $f(t)=\sum_{i=l_{1}}^{l_{2}} a_{i} t^{i}$ with $a_{l_{1}}, a_{l_{2}} \neq 0$ and minimal $\ell(f)$. Choose $m \in \mathbb{Z}$ such that $x^{m} t^{q} f(t) \in \mathcal{I}_{m}$. By (2.1), we have

$$
\begin{aligned}
{\left[x^{m} t^{q} f(t), x^{n} t^{q+j}\right]=} & x^{m+n} t^{q+j}\left((n q-m q-m j) f(t)+n t f^{\prime}(t)\right), \\
& \forall n \in \mathbb{Z}, j \in \mathbb{Z}_{+} .
\end{aligned}
$$

If $m=0$, by taking $n=1, j=0$ in $(2.3)$ we have $x^{1} t^{q}\left(q f(t)+t f^{\prime}(t)\right) \in \mathcal{I}_{1}$. Note that the coefficient of $t^{-q}$ in $f(t)$ is 0 when $-q \in \mathbb{N}$, hence $q f(t)+t f^{\prime}(t) \neq 0$. Moreover, $q f(t)+t f^{\prime}(t)$ is also strange, since the nonzero terms in $f(t)$ and in $q f(t)+t f^{\prime}(t)$ are the same but with different coefficients. Replacing $f(t)$ with $q f(t)+t f^{\prime}(t)$, if necessary, we may assume that $m \neq 0$. By computing $\left[\left[x^{m} t^{q} f(t), x^{1} t^{q}\right], x^{-1} t^{q}\right] \in \mathcal{I}_{m}$, we get $x^{m} t^{q}\left((3 q+1) t f^{\prime}(t)+t^{2} f^{\prime \prime}(t)\right) \in \mathcal{I}_{m}$. Set

$$
\begin{aligned}
g_{j}(t) & =\left(3 q j+j^{2}\right) f(t)-\left((3 q+1) t f^{\prime}(t)+t^{2} f^{\prime \prime}(t)\right) \\
& =\sum_{i=l_{1}}^{l_{2}}\left(\left(3 q j+j^{2}\right)-\left(3 q i+i^{2}\right)\right) a_{i} t^{i}, \quad \forall l_{1} \leqslant j \leqslant l_{2},
\end{aligned}
$$

then $x^{m} t^{q} g_{j}(t) \in \mathcal{I}_{m}$ and $\ell\left(g_{j}\right)<\ell(f)$. By the choice of $f$, we have $g_{j}=0$ for all $l_{1} \leqslant j \leqslant l_{2}$. This forces $f(t)=a_{l_{1}} t^{l_{1}}+a_{l_{2}} t^{l_{2}}$ and $\left(3 q l_{2}+l_{2}^{2}\right)-\left(3 q l_{1}+l_{1}^{2}\right)=$ $\left(l_{2}-l_{1}\right)\left(3 q+l_{1}+l_{2}\right)=0$, yielding $3 q+l_{1}+l_{2}=0$. By taking $n=0$ in $(2.3)$, we have

$$
x^{m} t^{q+j} f(t)=x^{m} t^{q}\left(a_{k} t^{l_{1}+j}+a_{l} t^{l_{2}+j}\right) \in \mathcal{I}_{m}, \forall j \in \mathbb{N}, j \neq-q .
$$


Moreover, $a_{l_{1}} t^{l_{1}+j}+a_{l_{2}} t^{l_{2}+j}$ is not strange since $3 q+\left(l_{1}+j\right)+\left(l_{2}+j\right) \neq 0$ for all $j \in \mathbb{N}, j \neq-q$. If $q \neq-1$, we have $x^{n} t^{q+l_{1}+1} \in \mathcal{I}$ for some $n \in \mathbb{Z}$ and hence $x^{m} t^{q+l_{2}} \in \mathcal{I}$ since $l_{2} \geqslant l_{1}+1$, contradiction.

Thus $q=-1$ and $x^{n} t^{q+l_{1}+2} \in \mathcal{I}$ for some $n \in \mathbb{Z}$, which implies $x^{m} t^{q+l^{\prime}} \in \mathcal{I}$ for all $l^{\prime} \geqslant l_{1}+2$. This forces $l_{2}=l_{1}+1$, which together with $3 q+l_{1}+l_{2}=0$ gives $l_{1}=1$ and $l_{2}=2$. It is clear that $x^{n} t^{q+i} \notin \mathcal{I}$ for all $n \in \mathbb{Z}, i=0,1,2$ and $x^{n} t^{q+i} \in \mathcal{I}$ for all $n \in \mathbb{Z}, i \geqslant 3$. Without loss of generality, suppose $x^{m} t^{q}\left(t+a m t^{2}\right) \in \mathcal{I}$ for some $a \in \mathbb{C}^{*}$ and $m \in \mathbb{Z}^{*}$. Using (2.3) we deduce that $\widetilde{J_{a}} \subseteq \mathcal{I}$. Now we can easily get $k=1$, thus $\mathcal{I}=\widetilde{J_{a}}$. This completes the proof of (1).

Statements (2) and (3) follow easily from (1).

Now we recall some known results from the representation theory of the Virasoro algebra and the Heisenberg-Virasoro algebra. The Heisenberg-Virasoro algebra HVir is the Lie algebra with the basis $\left\{L_{n}, I(n), C_{D}, C_{D I}, C_{I} \mid n \in \mathbb{Z}\right\}$ subject to the Lie brackets given by

$$
\begin{gathered}
{\left[L_{m}, L_{n}\right]=(n-m) L_{m+n}+\delta_{m,-n} \frac{m^{3}-m}{12} C_{D},} \\
{\left[L_{m}, I(n)\right]=n I(m+n)+\delta_{m,-n}\left(m^{2}+m\right) C_{D I},} \\
{[I(m), I(n)]=m \delta_{m,-n} C_{I},} \\
{\left[C_{D}, \mathrm{HVir}\right]=\left[C_{D I}, \mathrm{HVir}\right]=\left[C_{I}, \mathrm{HVir}\right]=0 .}
\end{gathered}
$$

The Virasoro algebra Vir is just the subalgebra of HVir spanned by $\left\{L_{n}, C_{D} \mid n \in\right.$ $\mathbb{Z}\}$.

Set $\mathcal{G}=\mathcal{B}(q)$, Vir, or HVir, we can define weight modules for $\mathcal{G}$ relative to the standard maximal toral subalgebra $Z+\mathbb{C} L_{0}$, where $Z$ is the center of $\mathcal{G}$ and $L_{0}=$ $q^{-1} L_{0,0}$ for $\mathcal{B}(q)$. Let $\mathcal{G}_{ \pm}$be the span of vectors in $\mathcal{G}$ with positive/negative eigenvalues of $\operatorname{ad}\left(L_{0}\right)$ respectively. In this paper, all weight modules are referred to the weight modules with central elements acting as scalars.

A weight $\mathcal{G}$-module $V$ is called highest/lowest weight if $V=U(\mathcal{G}) v$ for some nonzero $v$ with $\mathcal{G}_{ \pm} v=0$ respectively, is called Harish-Chandra if all its weight spaces are finitely dimensional (in case $\mathcal{G}=\mathcal{B}(q)$, it is usually called quasifinite since $\mathcal{B}(q)_{0}$ is infinite dimensional), is called uniformly bounded if all its weight spaces have dimension less than a fixed number, and is called a module of the intermediate series if all its weight spaces are no more than 1-dimensional. The notion of quasifinite modules was first used by V. Kac and A. Radul [KRa].

It is well known that any nontrivial irreducible uniformly bounded Vir-module is isomorphic to the irreducible submodule of $V(\alpha, \beta)$ for some $\alpha, \beta \in \mathbb{C}$. The modules $V(\alpha, \beta)$ all have a basis $\left\{v_{n} \mid n \in \mathbb{Z}\right\}$ with trivial central actions and

$$
L_{m} v_{n}=(\alpha+n+m \beta) v_{m+n} .
$$

The module $V(\alpha, \beta)$ is reducible if and only if $\alpha \in \mathbb{Z}$ and $\beta=0,1$. For convenience, we denote the unique nontrivial irreducible subquotient of $V(\alpha, \beta)$ by $V^{\prime}(\alpha, \beta)$. It is easy to see $V^{\prime}(0,0) \cong V^{\prime}(0,1)$. 
It was shown in [LZ2] that any nontrivial uniformly bounded irreducible HVirmodule is isomorphic to one of the irreducible modules $V^{\prime}(\alpha, \beta, F)$ for some $\alpha, \beta, F \in$ $\mathbb{C}$. The modules $V(\alpha, \beta, F)$ all have a basis $\left\{v_{n} \mid n \in \mathbb{Z}\right\}$ and the actions are given by

$$
L_{m} v_{n}=(\alpha+n+m \beta) v_{m+n}, \quad I(m) v_{n}=F v_{m+n}, \forall m, n \in \mathbb{Z}
$$

and $C_{D}, C_{I}, C_{D I}$ all act trivially. The module $V(\alpha, \beta, F)$ is reducible if and only if $F=0, \alpha \in \mathbb{Z}$ and $\beta=0,1$. We denote the corresponding unique nontrivial irreducible subquotient module by $V^{\prime}(\alpha, \beta, F)$. It is clear that $V^{\prime}(0,0,0) \cong V^{\prime}(0,1,0)$.

Note that $\mathcal{B}(q) / I_{1} \cong$ Vir for all $q \in \mathbb{C}^{*}$ and that if $-2 q \in \mathbb{N}$ then $\mathcal{B}(q) / I_{1}^{\prime} \cong$ $\left(\mathcal{B}(q) / I_{1}\right) \oplus\left(\mathbb{C} L_{0,-2 q}\right)$, where $\mathbb{C} L_{0,-2 q}$ is the 1-dimensional trivial center; $\mathcal{B}(-1) / I_{2} \cong$ $\mathrm{HVir} /\left(\mathbb{C} C_{D I}+\mathbb{C} C_{I}\right)$ and $\mathcal{B}(-1) / I_{2}^{\prime} \cong\left(\mathcal{B}(-1) / I_{2}\right) \oplus\left(\mathbb{C} L_{0,2}\right)$, where $\mathbb{C} L_{0,2}$ is the 1dimensional trivial center. We now define some $\mathcal{B}(q)$-modules:

(1). $V=V(\alpha, \beta, 0,0)$ for all $q \neq 0: I_{1} V=0$ and $V \cong V(\alpha, \beta)$ as $\mathcal{B}(q) / I_{1}$-modules;

(2). $V=V(\alpha, \beta, K, 0)$ for $-2 q \in \mathbb{N}: I_{1}^{\prime} V=0, V \cong V(\alpha, \beta)$ as $\mathcal{B}(q) / I_{1}$-modules and the trivial center $L_{0,-2 q}$ acts as a scalar $K$;

(3). $V=V(\alpha, \beta, K, F)$ for $q=-1: I_{2}^{\prime} V=0, V \cong V(\alpha, \beta, F)$ as $\mathcal{B}(q) / I_{2}$-modules and the trivial center $L_{0,2}$ acts as a scalar $K$.

Note that the above modules were defined in [SXX1] in a different approach. It is easy to see that $V(\alpha, \beta, K, F)$ is reducible if and only if $F=0, \alpha \in \mathbb{Z}$ and $\beta=0,1$. We denote the unique infinite-dimensional irreducible subquotient by $V^{\prime}(\alpha, \beta, K, F)$. When $-2 q \in \mathbb{N}$, the module $V(\alpha, \beta, K, 0)$ has a unique 1 -dimensional subquotient for any $\alpha \in \mathbb{Z}, \beta \in\{0,1\}$, denoted by $T(\alpha, \beta, K)$. Since $T(\alpha, \beta, K)$ is independent of $\alpha, \beta$ up to isomorphisms, we simply denote $T(K)=T(\alpha, \beta, K)$. We will show in the next section that $V^{\prime}(\alpha, \beta, K, F)$ and $T(K)$ exhaust all irreducible uniformly bounded $\mathcal{B}(q)$-modules.

3. Irreducible quasifinite modules. In this section we assume that $V$ is a nontrivial irreducible uniformly bounded $\mathcal{B}(q)$-module. Then there exists $\alpha \in \mathbb{C}$ such that $V$ admits a weight space decomposition $V=\oplus_{n \in \mathbb{Z}} V_{n}$ with

$$
V_{n}=\left\{v \in V \mid t^{q} v=q(\alpha+n) v\right\} .
$$

For convenience, we first introduce the concept of quasi-ideals of the polynomial ring $\mathbb{C}[t]$.

Definition 2. For any $k \in \mathbb{N}$, a subspace $K$ of $\mathbb{C}[t]$ is said to be a $k$-quasi-ideal of $\mathbb{C}[t]$ if $t^{k+i} K \subseteq K$ for all $i \in \mathbb{Z}_{+}$.

We can characterize $k$-quasi-ideals in $\mathbb{C}[t]$ as follows.

Lemma 3. Let $K$ be a nonzero subspace of $\mathbb{C}[t]$. Then $K$ is a $k$-quasi-ideal of $\mathbb{C}[t]$ if and only if there exists a monic polynomial $h(t) \in \mathbb{C}[t]$ such that $t^{k} h(t) \mathbb{C}[t] \subseteq$ $K \subseteq h(t) \mathbb{C}[t]$. In this case, we also say that $K$ is generated by $h(t)$ if further $h(t)$ is of minimal degree among such polynomials.

Proof. If a subspace $K$ satisfies $t^{k} h(t) \mathbb{C}[t] \subseteq K \subseteq h(t) \mathbb{C}[t]$ for some polynomial $h(t) \in \mathbb{C}[t]$, then we have

$$
t^{k+i} K \subseteq t^{k+i} h(t) \mathbb{C}[t] \subseteq t^{k} h(t) \mathbb{C}[t] \subseteq K, \forall i \in \mathbb{Z}_{+},
$$

that is, $K$ is a $k$-quasi-ideal of $\mathbb{C}[t]$.

Now suppose that $K$ is a nonzero subspace of $\mathbb{C}[t]$ such that $t^{k+i} K \subseteq K$ for all $i \in$ $\mathbb{Z}_{+}$. Let $h(t)$ be the greatest common divisor of polynomials in $K$, then $K \subseteq h(t) \mathbb{C}[t]$. 
By Bézout's Theorem of polynomials, there are polynomials $f_{1}(t), f_{2}(t), \ldots, f_{l}(t) \in K$ and $u_{1}(t), \ldots, u_{l}(t) \in \mathbb{C}[t]$ such that

$$
h(t)=u_{1}(t) f_{1}(t)+u_{2}(t) f_{2}(t)+\cdots+u_{l}(t) f_{l}(t) .
$$

Noticing that $t^{k+i} K \subseteq K$ for all $i \in \mathbb{Z}_{+}$, we see that $t^{k+i} u_{j}(t) f_{j}(t) \in K$ for all $i \in \mathbb{Z}_{+}$ and $1 \leqslant j \leqslant l$. Thus we have $t^{k+i} h(t) \in K$ for all $i \in \mathbb{Z}_{+}$, i.e., $t^{k} h(t) \mathbb{C}[t] \subseteq K$, as desired.

Lemma 4. There exists $k \in \mathbb{N}$ such that $\left(I_{k}+\mathbb{C} C\right) V=0$.

Proof. Note that $V$ can be viewed as a uniformly bounded module over the Virasoro algebra $\operatorname{span}\left\{C, L_{m, 0}: m \in \mathbb{Z}\right\}$. From the representation theory of the Virasoro algebra, we have $C V=0$ and there exists $p \in \mathbb{N}$ such that $\operatorname{dim} V_{n} \leqslant p$ for all $n \in \mathbb{Z}$.

Claim 1. For any $m, n_{0} \in \mathbb{Z}$ with $m \neq 0$, there is a nonzero 2-quasi-ideal $K_{m}$ so that $\left(x^{m} t^{q} K_{m}\right) V_{n_{0}}=0$.

Choose a basis $\left\{v_{1}, v_{2}, \cdots, v_{r}\right\}$ of $V_{n_{0}}$, where $0 \leqslant r \leqslant p$. Consider the following linear map

$$
\varphi_{m}: \quad \mathbb{C}[t] \rightarrow V_{n_{0}+m}^{r}=V_{n_{0}+m} \oplus V_{n_{0}+m} \oplus \cdots \oplus V_{n_{0}+m}
$$

defined by $\varphi_{m}(f)=\left(\left(x^{m} t^{q} f(t)\right) v_{1},\left(x^{m} t^{q} f(t)\right) v_{2}, \ldots,\left(x^{m} t^{q} f(t)\right) v_{r}\right)$ for any $f \in \mathbb{C}[t]$. Then for any $f(t) \in \operatorname{ker}\left(\varphi_{m}\right)$, we have $\left(x^{m} t^{q} f(t)\right) V_{n_{0}}=0$ and hence

$$
\begin{aligned}
0 & =\left[x^{m} t^{q} f(t), x^{0} t^{q+i}\right] V_{n_{0}}=-m(q+i)\left(x^{m} t^{q+i} f(t)\right) V_{n_{0}}, \forall i \in \mathbb{Z}_{+}, \\
0 & =\left[(q+i) x^{m} t^{q+i} f(t), x^{0} t^{q+j}\right] V_{n_{0}} \\
& =-m(q+i)(q+j)\left(x^{m} t^{q+i+j} f(t)\right) V_{n_{0}}, \forall i, j \in \mathbb{Z}_{+} .
\end{aligned}
$$

We have $t^{i} f(t) \in \operatorname{ker}\left(\varphi_{m}\right)$ for $f \in \operatorname{ker}\left(\varphi_{m}\right)$ and $i \in \mathbb{Z}_{+}$if $q \neq-1$; and $t^{i+2} f(t) \in$ $\operatorname{ker}\left(\varphi_{m}\right)$ for $f \in \operatorname{ker}\left(\varphi_{m}\right)$ and $i \in \mathbb{Z}_{+}$if $q=-1$. So $K_{m}=\operatorname{ker}\left(\varphi_{m}\right)$ is a nonzero 2-quasi-ideal of $\mathbb{C}[t]$.

Let $K_{m}$ be generated by the monic polynomial $P_{m}(t)$ (assuming that $n_{0}$ is fixed). By Lemma 3, we have $K_{m} \subseteq P_{m} \mathbb{C}[t]$ and $\operatorname{deg} P_{m} \leqslant \operatorname{dim}\left(\mathbb{C}[t] / K_{m}\right) \leqslant \operatorname{dim}\left(V_{n_{0}+m}^{r}\right) \leqslant$ $p^{2}$.

Claim 2. For any $n \in \mathbb{Z}$, there is a 2 -quasi-ideal $R_{n}$ so that $\left(x^{m} t^{q} R_{n}\right) V_{n}=0$ for all $m \in \mathbb{Z}^{*}$.

Fix any $n_{0} \in \mathbb{Z}$. Take arbitrary $m, n \in \mathbb{Z}$ with $m n(m+n) \neq 0$. We have monic polynomials $P_{m}$ and $P_{n}$ with respect to $n_{0}$. Applying (2.1) with $f=t^{q+i} P_{m}$, $g=t^{q+j} P_{n}$, to $V_{n_{0}}$, we have

$$
\begin{gathered}
P_{m+n} \mid t^{i+j}\left[\left((n i-m j) P_{m} P_{n}\right)+\left((n q-m q) P_{m} P_{n}+n t P_{m}^{\prime} P_{n}-m t P_{m} P_{n}^{\prime}\right)\right], \\
\forall i, j \geqslant 2 .
\end{gathered}
$$

Taking $(i, j)=(2,3)$ and $(i, j)=(3,2)$, we deduce that

$$
P_{m+n} \mid t^{5} P_{m} P_{n} \quad \text { and } \quad P_{m+n} \mid t^{5}\left((n q-m q) P_{m} P_{n}+n t P_{m}^{\prime} P_{n}-m t P_{m} P_{n}^{\prime}\right) .
$$

Using (3.3), we can inductively deduce that

$$
P_{j+2} \mid t^{5 j} P_{1}^{j} P_{2} \quad \text { and } \quad P_{-(j+2)} \mid t^{5 j} P_{-1}^{j} P_{-2}
$$


for any $j \in \mathbb{N}$. Recalling the fact $\operatorname{deg} P_{m} \leqslant p^{2}$, we have $P_{m} \mid\left(t P_{1} P_{2} P_{-1} P_{-2}\right)^{p^{2}}$ and hence $t^{2}\left(t P_{1} P_{2} P_{-1} P_{-2}\right)^{p^{2}} \mathbb{C}[t] \subseteq \bigcap_{j \in \mathbb{Z}^{*}} K_{j}$. Set

$$
R_{n_{0}}=\bigcap_{j \in \mathbb{Z}^{*}} K_{j}=\left\{f(t) \in \mathbb{C}[t] \mid\left(x^{j} t^{q} f(t)\right) V_{n_{0}}=0, \quad \forall j \in \mathbb{Z}^{*}\right\}
$$

which is easily proved to be a 2-quasi-ideal.

Let $R_{n}$ be generated by the monic polynomial $Q_{n}(t)$. Then

$$
\operatorname{deg} Q_{n}(t) \leqslant \operatorname{deg}\left(t^{2}\left(t P_{1} P_{2} P_{-1} P_{-2}\right)^{p^{2}}\right) \leqslant p^{2}\left(4 p^{2}+1\right)+2 \leqslant 7 p^{4} .
$$

Claim 3. $Q_{k}(t)$ is a power of $t$ for any $k \in \mathbb{Z}$.

Choose any $m, k \in \mathbb{Z}$ with $m \neq 0,-1$. Let $h(t)$ be the least common multiple of $Q_{k}(t), Q_{m+k}(t)$ and $Q_{m+k+1}(t)$, then $t^{2} h(t) \in R_{k} \cap R_{m+k} \cap R_{m+k+1}$. By taking $f(t)=t^{q}$ and $g(t)=t^{q+2} h(t)$ in $(2.1)$, we have

$$
\left[x^{m} t^{q}, x^{n} t^{q+2} h(t)\right]=x^{m+n} t^{q+2}\left((n q-m q-2 m) h(t)-m t h^{\prime}(t)\right) .
$$

Applying the above equation to $V_{k}$, we get

$$
x^{m+n} t^{q+3} h^{\prime}(t) V_{k}=0, \quad \forall n \neq 0,-m .
$$

Replacing $m$ with $m+1$, similarly we get

$$
x^{m+n+1} t^{q+3} h^{\prime}(t) V_{k}=0, \quad \forall n \neq 0,-m-1 .
$$

Combining the above two formulas, we deduce that

$$
x^{n} t^{q+3} h^{\prime}(t) V_{k}=0, \quad \forall n \in \mathbb{Z}^{*},
$$

which yields $t^{3} h^{\prime}(t) \in R_{k}$ and $Q_{k} \mid t^{3} h^{\prime}(t)$.

Suppose that there exist $a \in \mathbb{C}^{*}$ and $n \in \mathbb{Z}$ such that $(t-a) \mid Q_{n}(t)$. We can choose $d \in \mathbb{N}$ and $k \in \mathbb{Z}$ such that $(t-a)^{d} \mid Q_{k}$ and $(t-a)^{d+1} \nmid Q_{l}$ for any $l \in \mathbb{Z}$. Then by the previous result, we have $Q_{k} \mid t^{3} h^{\prime}(t)$ and hence $(t-a)^{d} \mid t^{3} h^{\prime}(t)$, where $h(t)$ is defined as before. On the other hand, we have $(t-a)^{d+1} \nmid h(t)$ and $(t-a)^{d} \nmid h^{\prime}(t)$ by the definition of $h(t)$, contradiction! Thus any $Q_{n}(t)$ must be a power of $t$.

Since all $\operatorname{deg} Q_{n} \leqslant 7 p^{4}$, there exists $k_{0} \in \mathbb{N}$ such that $\left(x^{m} t^{q+i}\right) V_{n}=0$ for all $m \in \mathbb{Z}^{*}, n \in \mathbb{Z}$ and $i \geqslant k_{0}$. Then by the Lie bracket (2.1), we get

$$
0=\left[x^{m} t^{q+k_{1}}, x^{-m^{q}} t^{q+k_{2}}\right] V_{n}=-m\left(2 q+k_{1}+k_{2}\right) x^{0} t^{q+k_{1}+k_{2}} V_{n},
$$

for all $m \in \mathbb{Z}^{*}, n \in \mathbb{Z}$ and $k_{1}, k_{2} \geqslant k_{0}$. Then there exists $k \in \mathbb{N}$ such that

$$
\left(x^{m} t^{q+i}\right) V_{n}=0, \forall m \in \mathbb{Z}, n \in \mathbb{Z} \text { and } i \geqslant k,
$$

that is, $\left(I_{k}+\mathbb{C} C\right) V=0$, as desired.

THEOREM 5. Let $V$ be an irreducible uniformly bounded $\mathcal{B}(q)$-module where $q \in$ $\mathbb{C}^{*}$. Then $V$ is a module of intermediate series. More precisely, $V$ is isomorphic to $T(K)$ or $V^{\prime}(\alpha, \beta, K, F)$ for suitable $\alpha, \beta, K, F \in \mathbb{C}$.

Proof. If $I_{0}^{\prime} V=0$, then $V \cong T(K)$ for some $K \in \mathbb{C}$. Now we suppose that $I_{0}^{\prime} V \neq 0$. 
By Lemma 4 , there exists $k \in \mathbb{Z}_{+}$such that $I_{k+1}^{\prime} V=0$ and $V$ can be viewed as an irreducible uniformly bounded module over $\mathcal{B}(q)^{(k)}=\mathcal{B}(q) / I_{k+1}^{\prime}$. We still denote by $L_{m, i}$ its image in $\mathcal{B}(q)^{(k)}$.

If $k=0$, then $\mathcal{B}(q)^{(0)}$ is isomorphic to $\operatorname{Vir} \oplus \delta_{-2 q, \mathbb{N}} \mathbb{C} L_{0,-2 q}$ and $V \cong V^{\prime}(\alpha, \beta)$ for some $\alpha, \beta \in \mathbb{C}$ as Vir-modules with action of the center $L_{0,-2 q}$ being an arbitrary scalar. In this case, $V \cong V^{\prime}(\alpha, \beta, K, 0)$ for some $K \in \mathbb{C}$ as $\mathcal{B}(q)$-modules.

If $k=1$ and $q=-1$, then $\mathcal{B}(q)^{(1)}$ is isomorphic to a one-dimensional trivial central extension of $\mathrm{HVir} /\left(\mathbb{C} C_{D I} \oplus \mathbb{C} C_{I}\right)$ and $V \cong V^{\prime}(\alpha, \beta, F)$ for some $\alpha, \beta, F \in \mathbb{C}$ as HVir-modules with action of the trivial center being an arbitrary scalar. In this case, $V \cong V^{\prime}(\alpha, \beta, K, F)$ for some $K \in \mathbb{C}$ as $\mathcal{B}(q)$-modules.

Now we suppose that $k \geqslant 1$ if $q \neq-1$, and $k \geqslant 2$ if $q=-1$. Denote

$$
H=\operatorname{span}_{\mathbb{C}}\left\{L_{m, k} \mid m \in \mathbb{Z},(m, k) \neq(0,-2 q)\right\} \subseteq \mathcal{B}(q)^{(k)} .
$$

Note that $L_{m, i}=0$ in $\mathcal{B}(q)^{(k)}$ for all $m, i \in \mathbb{Z}$ with $i>k$ and $(m, i) \neq(0,-2 q)$. Let $p \in \mathbb{N}$ be such that $\operatorname{dim} V_{n} \leq p$ for all $n \in \mathbb{Z}$.

Claim 1. For any $1 \leqslant l \leqslant k$, there is $a_{l} \in \mathbb{C}$ such that $\left(L_{0, l}-a_{l}\right)^{p} V=0$.

Take any $1 \leqslant l \leqslant k$ and $w \in V$. Suppose that we have $r \in \mathbb{N}$ and $a \in \mathbb{C}$ such that $\left(L_{0, l}-a\right)^{r} w=0$. We denote $w_{i}=\left(L_{0, l}-a\right)^{i} w$ for $0 \leqslant i \leqslant r$. Then $\left(L_{0, l}-a\right)^{r} L_{m, j} w_{i}=0$ for all $0 \leqslant i \leqslant r$ and $j \geqslant k+1-l$, since $\left[L_{0, l}, L_{m, j}\right]=0$.

Now take $0 \leqslant i \leqslant r$ and $j \geqslant k+1-l$. Suppose that $\left(L_{0, l}-a\right)^{(s+1) r} L_{m, j-s^{\prime}} w_{i}=0$ for all $0 \leqslant s^{\prime} \leqslant s$ for some $0 \leqslant s \leqslant k-l$. Then we have

$$
\begin{aligned}
& \left(L_{0, l}-a\right)^{(s+2) r} L_{m, j-s-1} w_{i} \\
= & \left(L_{0, l}-a\right)^{(s+2) r-1}\left[L_{0, l}, L_{m, j-s-1}\right] w_{i}+\left(L_{0, l}-a\right)^{(s+2) r-1} L_{m, j-s-1} w_{i+1} \\
= & \left(L_{0, l}-a\right)^{(s+2) r-1} L_{m, j-s-1} w_{i+1}=\cdots=\left(L_{0, l}-a\right)^{(s+1) r+i} L_{m, j-s-1} w_{r}=0 .
\end{aligned}
$$

By induction, we have

$$
\left(L_{0, l}-a\right)^{r(k+2-l)} L_{m, j} w=0, \quad \forall 0 \leqslant j \leqslant k .
$$

Then we conclude that for all $x \in U\left(\mathcal{B}(q)^{(k)}\right)$ there is $r^{\prime} \in \mathbb{N}$ such that $\left(L_{0, l}-a\right)^{r^{\prime}} x w=$ 0 .

Fix any nonzero weight space $V_{n_{0}}$ for some $n_{0}$. Then there exists nonzero $v \in V_{n_{0}}$ such that $\left(L_{0, l}-a_{l}\right) v=0$ for some $a_{l} \in \mathbb{C}$. Take $x_{1}, \cdots, x_{s} \in U\left(\mathcal{B}(q)^{(k)}\right)_{n-n_{0}}$ such that $\left\{x_{1} v, \cdots, x_{s} v\right\}$ forms a basis of $V_{n}$. Then by the discussion in last paragraph, there exists $r_{n} \in \mathbb{N}$ such that $\left(L_{0, l}-a_{l}\right)^{r_{n}} x_{i} v=0$ for all $1 \leqslant i \leqslant s$, or, $\left(L_{0, l}-a_{l}\right)^{r_{n}} V_{n}=0$. Since $\operatorname{dim} V_{n} \leqslant p$ for some $p \in \mathbb{N}$, hence $\left(L_{0, l}-a_{l}\right)^{p} V_{n}=0$ for all $n \in \mathbb{Z}$, i.e., $\left(L_{0, l}-a_{l}\right)^{p} V=0$.

Claim 2. $H V=0$.

Fix any $1 \leqslant l \leqslant k$ with $l \neq-q$, there is $r \in \mathbb{Z}_{+}$such that $\left(L_{0, l}-a_{l}\right)^{r+1} V=0$ and $u=\left(L_{0, l}-a_{l}\right)^{r} v \neq 0$ for some $v \in V$. We have

$$
\begin{aligned}
0 & =L_{m, k-l}\left(L_{0, l}-a_{l}\right)^{r+1} v=\sum_{j=0}^{r}\left(L_{0, l}-a_{l}\right)^{j}\left[L_{m, k-l}, L_{0, l}-a_{l}\right]\left(L_{0, l}-a\right)^{r-j} v \\
& =-m(r+1)(q+l) L_{m, k}\left(L_{0, l}-a_{l}\right)^{r} v=-m(r+1)(q+l) L_{m, k} u,
\end{aligned}
$$

which gives $L_{m, k} u=0$ for all $m \in \mathbb{Z}^{*}$. 
On the other hand, if there exists $0 \leqslant i<r+1$ such that $L_{m_{1}, k} \cdots L_{m_{i}, k}\left(L_{0, l}-\right.$ $\left.a_{l}\right)^{r+1-i} V=0$ for all $m_{1}, m_{2}, \cdots, m_{i} \in \mathbb{N}$, then

$$
\begin{aligned}
0 & =L_{m_{i+1}, k-l} L_{m_{1}, k} \cdots L_{m_{i}, k}\left(L_{0, l}-a_{l}\right)^{r+1-i} V \\
& =L_{m_{1}, k} \cdots L_{m_{i}, k} L_{m_{i+1}, k-l}\left(L_{0, l}-a_{l}\right)^{r+1-i} V \\
& =L_{m_{1}, k} \cdots L_{m_{i}, k} \sum_{j=0}^{r-i}\left(L_{0, l}-a_{l}\right)^{j}\left[L_{m_{i+1}, k-l}, L_{0, l}-a_{l}\right]\left(L_{0, l}-a_{l}\right)^{r-i-j} V \\
& =-m_{i+1}(r+1-i)(q+l) L_{m_{1}, k} \cdots L_{m_{i}, k} L_{m_{i+1}, k}\left(L_{0, l}-a_{l}\right)^{r-i} V,
\end{aligned}
$$

which gives

$$
L_{m_{1}, k} \cdots L_{m_{i}, k} L_{m_{i+1}, k}\left(L_{0, l}-a_{l}\right)^{r-i} V=0, \quad \forall m_{i+1} \in \mathbb{N} .
$$

Then using induction based on the previous discussion and by the fact $\left(L_{0, l}-\right.$ $\left.a_{l}\right)^{r+1} V=0$, we can deduce that

$$
L_{m_{1}, k} \cdots L_{m_{r+1}, k} V=0, \quad \forall m_{1}, \cdots m_{r+1} \in \mathbb{N} .
$$

If $k=-2 q$, for the nonzero vector $u \in V$ we have $L_{m, k} u=0$ for all $m \in \mathbb{Z}^{*}$, that is $H u=0$. Thus $H V=0$ since $H$ is an ideal of $\mathcal{B}(q)^{(k)}$ and $V$ is an irreducible $\mathcal{B}(q)^{(k)}$-module.

If $k \neq-2 q$, then there exists $s \in \mathbb{N}$ such that $L_{1, k}^{s} V=0$ and $L_{1, k}^{s-1} V \neq 0$ by the above discussion, then

$$
0=L_{-1,0} L_{1, k}^{s} V=\left[L_{-1,0}, L_{1, k}^{s}\right] V=s(k+2 q) L_{0, k} L_{1, k}^{s-1} V .
$$

Recall that we have $\left(L_{0, k}-a_{k}\right)^{p} V=0$ from Claim 1 , so $a_{k}=0$ and hence $L_{0, k}^{p} V=0$. In particular, we have $L_{0, k}^{p} u=0$. Let $r^{\prime} \in \mathbb{N}$ be such that $L_{0, k}^{r^{\prime}} u=0$ and $u_{0}=$ $L_{0, k}^{r^{\prime}-1} u \neq 0$. Now we have

$$
L_{0, k} u_{0}=0 \text { and } L_{m, k} u_{0}=L_{m, k} L_{0, k}^{r^{\prime}-1} u=L_{0, k}^{r^{\prime}-1} L_{m, k} u=0 \quad \forall m \in \mathbb{Z}^{*},
$$

i.e., $H u_{0}=0$. This implies $H V=0$.

Thus we have obtained that $I_{k}^{\prime} V=0$. By induction on $k$, we can deduce that $I_{1}^{\prime} V=0$ if $q \neq-1$ and $I_{2}^{\prime} V=0$ if $q=-1$. Our result follows from the discussion at the beginning of the proof.

REMARK 6. In fact, we can also define the ideals $I_{k}$ of $\mathcal{B}(0)$ for all $k \in \mathbb{Z}_{+}$ similarly. Then using the same methods as those in the proofs of Lemma 4 and Theorem 5, we can show that $I_{1} V=0$ for any irreducible uniformly bounded $\mathbb{Z}$ graded $\mathcal{B}(0)$-module, where the gradation is induced from the gradation (2.2). Since $\mathcal{B}(0) / I_{1}$ is isomorphic to an infinite-dimensional Heisenberg algebra, by the results of $[\mathrm{C}]$ we can determine all irreducible uniformly bounded $\mathbb{Z}$-graded $\mathcal{B}(0)$-module; in particular, the homogeneous spaces of such modules are no more than 1-dimensional.

Note that the simplest case of Theorem 6 recovers Theorem 1.5 in [SXX1] which classified all irreducible modules of intermediate series over $\mathcal{B}(q)$ whose proof was based on about ten pages of computations. When we were revising our paper, we noticed the paper [SXX2], where Theorem 5 for the special case $q=1$ is obtained independently. Right away, we sent the preprint of the present paper to the authors of [SXX2] on Thursday, Jan 10, 2013. 
Combining the above theorem with Theorem 1.3 in [SXX1], now we give a complete classification of irreducible quasifinite modules over $\mathcal{B}(q)$.

Theorem 7. Let $q \in \mathbb{C}^{*}$. Any irreducible quasifinite module over $\mathcal{B}(q)$ is isomorphic to an irreducible highest weight module or an irreducible lowest weight module, or a module of the form $T(K)$ or $V^{\prime}(\alpha, \beta, K, F)$ for suitable $\alpha, \beta, K, F \in \mathbb{C}$.

Recently, the irreducible unitary modules for the algebras $\mathcal{B}(q)$ were classified in $[\mathrm{CG}]$.

For any $\beta \in \mathbb{C}$, let $\mathcal{L}(\beta)$ be the Lie algebra which is the semi-direct product of the Virasoro algebra and one of its intermediate series module $V(0, \beta)$; more precisely, $\mathcal{L}(\beta)$ has a basis $\left\{L_{n}, L_{n}^{\prime}, C \mid n \in \mathbb{Z}\right\}$ and the Lie brackets are defined as

$$
\begin{gathered}
{\left[L_{m}, L_{n}\right]=(n-m) L_{m+n}+\delta_{m+n, 0} \frac{m^{3}-m}{12} C,} \\
{\left[L_{m}, L_{n}^{\prime}\right]=(n+m \beta) L_{m+n}^{\prime}+\delta_{\beta,-1} \delta_{m+n, 0} \frac{m^{3}-m}{12} C,} \\
{\left[L_{m}^{\prime}, L_{n}^{\prime}\right]=0}
\end{gathered}
$$

where $m, n \in \mathbb{Z}$ and $C$ is central. It is clear that $\mathcal{L}(0)$ and $\mathcal{L}(-1)$ are isomorphic to HVir / $\left(\mathbb{C} C_{D I} \oplus \mathbb{C} C_{I}\right)$ and $W(2,2)$ respectively; the classification of irreducible HarishChandra modules for these algebras were given in [LZ2] and [GLZ2] respectively.

Note that $\mathcal{L}(\beta) \cong \mathcal{B}(q) / I_{2}$ where $\beta=-1-1 / q$. From Theorem 5 we can have

Corollary 8. Let $\beta \in \mathbb{C} \backslash\{0,-1\}$, and $H$ be the ideal of $\mathcal{L}(\beta)$ spanned by $L_{n}^{\prime}$ for $n \in \mathbb{Z}$ if $\beta \neq 1$ or $n \in \mathbb{Z}^{*}$ if $\beta=1$. Assume that $V$ is an irreducible uniformly bounded module over $\mathcal{L}(\beta)$. Then $H V=0$ and $V$ is of intermediate series.

Acknowledgements. The research was carried out during the visit of the first and second authors at Wilfrid Laurier University. They gratefully acknowledge the support (a short term grant) and hospitality of Wilfrid Laurier University. The first author is partially supported by the Recruitment Program of Global Youth Experts of China, by the start-up funding from University of Science and Technology of China, by NSF of China (Grant 11401551). The second author is partially supported by NSF of China (Grant 11101380, 11471294) and the CSC of China (Grant 2010841037). The third author is partially supported by NSF of China (Grant 11271109) and NSERC. We would also like to thank the referee for helpful comments and suggestions.

\section{REFERENCES}

[C] V. CHARI, Integrable representations of affine Lie-algebras, Invent. Math., 85 (1986), pp. 317-335.

[CG] H. Chen And X. Guo, Unitary Harish-Chandra modules over Block type Lie algebras $\mathcal{B}(q)$, J. Lie Theory, 23:3 (2013), pp. 827-836.

[CP] V. Chari And A. PRessley, Unitary representations of the Virasoro algebra and a conjecture of Kac, Compositio Math., 67 (1988), pp. 315-342.

[DZ] D. Z. DJokovic AND K. ZhaO, Derivations, isomorphisms and second cohomology of generalized Block algebras, Algebra Colloq., 3 (1996), pp. 245-272.

[GLZ1] X. Guo, R. Lu, AND K. Zhao, Classification of irreducible Harish-Chandra modules over generalized Virasoro algebras, Proc. Edinb. Math. Soci., 55 (2012), pp. 697-709. 
[GLZ2] X. Guo, R. Lu, And K. Zhao, Simple Harish-Chandra modules, intermediate series modules, and Verma modules over the loop-Virasoro algebra, Forum Math., 23 (2011), pp. 1029-1052.

[KR] V. G. KAC AND A. K. RAINA, Bombay lectures on highest weight representations of infinitedimensional Lie algebras, Advanced Series in Mathematical Physics, 2. World Scientific Publishing Co., Inc., Teaneck, NJ, 1987.

[KRa] V. G. KAC AND A. RADUL, Quasifinite highest weight modules over the Lie algebra of differential operators on the circle, Comm. Math. Phys., 157 (1993), pp. 429-457.

[LS] J. Li And Y. Su, Representations of the Schrödinger-Virasoro algebras, J. Math. Phys., 49 (2008), 053512, 14pp.

[LT1] W. Lin AND S. TAN, Nonzero level Harish-Chandra modules over the Virasoro-like algebra, J. Pure. Appl. Algebra, 204 (2006), pp. 90-105.

[LT2] W. LIN AND S. TAN, Graded modules over the q-analog Virasoro-like algebra, Algebr. Represent. Theory, 11 (2008), pp. 505-517.

[LZ1] R. Lu AND K. ZhaO, Classification of irreducible weight modules over higher rank Virasoro algebras, Adv. Math., 206 (2006), pp. 630-656.

[LZ2] R. LU AND K. ZHAO, Classification of irreducible weight modules over the twisted Heisenberg-Virasoro algebra, Commun. Contemp. Math., 12 (2010), pp. 183-205.

[M] O. Mathieu, Classification of Harish-Chandra modules over the Virasoro Lie algebra, Invent. Math., 107 (1992), pp. 225-234.

[S1] Y. Su, Classification of Harish-Chandra modules over the higher rank Virasoro algebras, Comm. Math. Phys., 240 (2003), pp. 539-551.

[S2] Y. Su, Quasifinite representations of a Lie algebra of Block type, J. Algebra, 276 (2004), pp. $117-128$.

[S3] Y. Su, Quasifinite representations of a family of Lie algebras of Block type, J. Pure Appl. Algebra, 192 (2004), pp. 293-305.

[SXX1] Y. Su, C. XIA, AND Y. XU, Quasifinite representations of a class of Block type Lie algebras $\mathcal{B}(q)$, J. Pure Appl. Algebra, 216 (2012), pp. 923-934.

[SXX2] Y. SU, C. XIA, AND Y. XU, Classification of quasifinite representations of a Lie algebra related to Block type, J. Algebra, 393 (2013), pp. 71-78.

[WT] Q. WANG AND S. TAN, Quasifinite modules of a Lie algebra related to Block type, J. Pure Appl. Algebra, 211 (2007), pp. 596-608. 
